\title{
Reactivation of old scars: inevitably sarcoid
}

\section{J S Sorabjee, R Garje}

Postgrad Med J 2005;81:60-61. doi: 10.1136/pgmj.2004.018796

Reactivation of pre-existing scars, as a manifestation of cutaneous sarcoidosis, is uncommon and the clinical significance of this odd symptom often remains unrecognised. In the appropriate setting a skin biopsy may serve to establish the diagnosis of sarcoidosis and avoid more invasive attempts at obtaining tissue. The case of a 72 year old man with remote reactivation of multiple cutaneous scars associated with a febrile illness, malaise, and hilar adenopathy is reported. A scar biopsy revealed multiple non-caseating granulomas and established the diagnosis of sarcoidosis. A short course of steroids was successful in causing regression of symptoms and cutaneous inflammation. Regression of inflammation in the scar provides a simple way to monitor response to treatment in this multisystem disease.

S arcoidosis, though uncommon, is being recognised with increasing frequency in India. ${ }^{1}$ Reactivation of old scars, as a manifestation of cutaneous sarcoidosis, is highly specific for sarcoidosis but very unusual. ${ }^{23}$ Clinicians unaware of the significance of this odd sign may tend to dismiss it lightly. Easy access to histopathology via a skin biopsy is useful in confirming the diagnosis. Regression of the lesions with treatment is useful in assessing the response in this multisystem disease.

\section{CASE REPORT}

A 72 year old man presented with a four week history of a low grade fever (temperature $<38.5^{\circ} \mathrm{C}$ ) associated with $5 \mathrm{~kg}$ of weight loss. He had been treated with antibiotics empirically by his family practitioner without benefit. Routine investigations including a complete blood count, erythrocyte sedimentation rate (ESR), urinalysis, and chest radiography were normal and he was therefore referred for further consultation. When seen five weeks after the onset of his fever he volunteered a history of swelling, redness, and itching associated with numerous scars that he had acquired almost 50 years previously during his career in professional cricket. Physical examination was entirely normal other than the presence of swelling and discoloration at the sites of four scars on his upper lip (fig 1), back, chest, and neck. In view of a suspicious hilar prominence on chest radiography, computed tomography of his thorax was done; this showed the presence of numerous non-necrotic lymph nodes in the mediastinum but without any lung lesions. Serum angiotensin converting enzyme concentrations were normal as were repeat examinations of his complete blood count, ESR, peripheral smear, and routine biochemistry. A Mantoux test to 5 and 10 tuberculin units was negative.

In view of the above clinical presentation a differential diagnosis of mediastinal tuberculosis, sarcoidosis, and lymphoma was considered. As no peripheral lymph nodes were palpable, a decision was made to perform a scar biopsy before proceeding with a mediastinal lymph node biopsy. This was obtained from the most active scar, which was present on the lower back. Histopathology revealed the presence of numerous non-caseating granulomas enmeshed within the fibrous tissue of the scar. Treatment was instituted with a short course of steroids; after this the patient's fever settled, he gained weight, and the visible inflammation in the scars regressed completely. Over a period of time the lesions seen on chest radiography regressed on steroid therapy, which was gradually withdrawn.

\section{DISCUSSION}

In India sarcoidosis is uncommon but has been recognised with increasing frequency in recent years. In most instances patients present with pulmonary involvement manifested by hilar adenopathy and interstitial lung lesions. Skin manifestations are relatively uncommon $(20 \%-35 \%)^{4}$ and occur in three broad groups-that is, erythema nodosum, infiltrative cutaneous sarcoidosis, and scar sarcoidosis. Scar sarcoidosis is itself rare $(2.9 \%)^{3}$ with few case reports worldwide and fewer from India. It is especially uncommon in the absence of other skin manifestations of sarcoidosis and takes the form of redness and reactivation of old cutaneous scars. Scar sarcoidosis may precede or accompany systemic sarcoidosis and may be the main manifestation of a relapse. ${ }^{5}$ Sarcoid isolated to scars may represent a more benign variant of sarcoidosis, and has been considered to be a systemic autoimmune disease. ${ }^{6}$

Reactivation of old scars appears to be highly specific for sarcoidosis and in this instance the positive skin biopsy mitigated the need for an invasive procedure in the form of a mediastinal lymph node biopsy. ${ }^{7}$

In addition to reactivation of scars obtained from previous wounds, scar sarcoidosis has been reported at the sites of previous intramuscular injections, tattoo scars, blood donation venepuncture sites, scars of herpes zoster, ${ }^{8}$ sarcoidosis on ritual scarification, ${ }^{9}$ and at the sites of allergen extracts for desensitisation. ${ }^{10}$ As in this patient a short course of steroids in moderate doses (30 mg per day in this instance) serves to suppress most of the symptoms of the disease, however in cases where scar sarcoidosis is the only manifestation of the disease, allopurinol in a dose of $300 \mathrm{mg}$ per day has been found to be

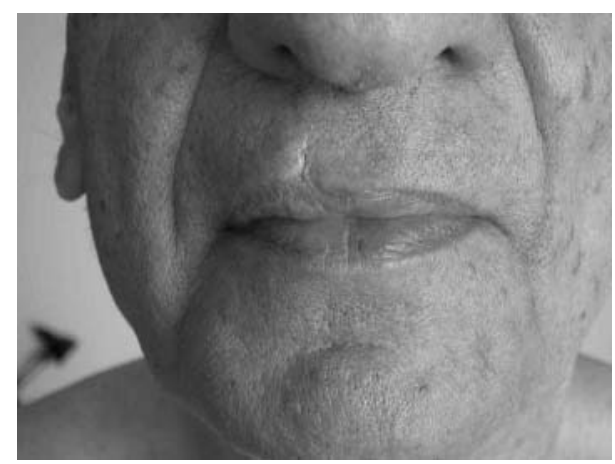

Figure 1 Small scar on patient's lip that had been quiescent for 30 years; this developed redness and swelling associated with the onset of sarcoidosis (published with patient's permission). 
effective. ${ }^{11}$ It is essential that patients complaining of inflammation at the sites of old scars should be examined and in the appropriate setting investigated for sarcoidosis.

\section{Authors' affiliations}

J S Sorabjee, R Garje, Department of Medicine, Bombay Hospital Institute of Medical Sciences, Bombay, India

Correspondence to: Dr Jehangir S Sorabjee, Department of Medicine, Bombay Hospital Institute of Medical Sciences, 12 New Marine Lines, Bombay 400020, Maharashtra State, India; jsorabjee@vsnl.com

Submitted 8 January 2004

Accepted 20 February 2004

\section{REFERENCES}

1 Murlidhar S, Handa S, Sharma VK, et al. Scar sarcoidosis-sparing post surgical scar. Indian Journal of Dermatology Venereology and Leprology 1999:65:279-80
2 Manz LA, Rodman OG. Reappearance of quiescent scars. Arch Dermatol 1993; 129:105, 108.

3 Yanardag H, Pamuk ON, Karayel T. Cutaneous involvement in sarcoidosis: analysis of the features in 170 patients. Respir Med 2003:97:978-82

4 Samtsov AV. Cutaneous sarcoidosis. Int J Dermatol 1992;31:385-91.

5 Chudomirova K, Velichkova L, Anavi B, et al. Recurrent sarcoidosis in skin scars accompanying systemic sarcoidosis. J Eur Acad Dermatol Venereol 2003; 17:360-1.

6 Morhenn VB, Smoller BR. Immunophenotyping of a sarcoidal granuloma in a scar, a manifestation of a possible autoimmune process. J Cutan Med Surg 1998:3:46-8.

7 Sarkar TK. Sarcoidosis diagnosed by biopsy of the scar. J Indian Med Assoc 1967;48:29-30.

8 Alabi GO, George AO. Cutaneous sarcoidosis and tribal scarifications in West Africa. Int J Dermatol 1989;28:29-31.

9 Corazza M, Bacilieri S, Strumia R. Post-herpes zoster scar sarcoidosis. Acta Dermatol Venereol 1999;79:95.

10 Healsmith MF, Hutchinson PE. The development of scar sarcoidosis at the site of desensitization injections. Clin Exp Dermatol 1992;17:369-7.

11 Pfau A, Stolz W, Karrer S, et al. Allopurinol in treatment of cutaneous sarcoidosis. Hautarzt 1998:49:216-8. 